\title{
Asperity Heating for Repair of Metal Contact RF MEMS Switches
}

\author{
Brian D. Jensen ${ }^{1}$, Kuangwei Huang ${ }^{1}$, Linda Chow $^{1}$, Kazuhiro Saitou ${ }^{1}$, John L. Volakis ${ }^{2}$, and Katsuo Kurabayashi ${ }^{1}$ \\ ${ }^{1}$ Department of Mechanical Engineering, University of Michigan, Ann Arbor, MI, 48105, USA \\ ${ }^{2}$ Department of Electrical Engineering, Ohio State University, Columbus, OH, 43210, USA
}

\begin{abstract}
We have experimentally observed the failure of metal contact RF MEMS switches due to a rapid rise in contact resistance during switching. We were able to repair the failed switches through heating the contact asperities by applying sufficient contact voltage. The data suggest the hypothesis that increasing contact resistance is caused by strain hardening of the contact surface. With this understanding, appropriate corrective measures can be taken to overcome failure, and suggestions for doing so are given in the paper.

Index Terms-RF MEMS switch reliability, failure
\end{abstract}

\section{INTRODUCTION}

Many examples of metal contact RF MEMS switches are presented in the literature (see, for example, [1], [2]). These have demonstrated the excellent performance typical of MEMS switches - high off-state impedance, low onstate impedance, excellent linearity, and low power consumption. In addition, metal contact switches offer significantly wider bandwidth compared to capacitive switches. However, they are in general less reliable than capacitive switches due to the complex interactions between deformation, current flow, and heating at the contact [3].

Several different failure mechanisms have been noted for metal contact switches, such as contact adhesion [4], melting, material transport [5], thermally-induced explosions of contact material [6], and increasing contact resistance [7]. We are currently performing modeling and experiments to better understand contact heating [8], [9]. We have observed severe increases in contact resistance as the switch cycles, leading to rapid failure of the switch. We demonstrate a method to repair failed switches by heating the contact by applying a contact voltage. Operating conditions are also presented which allow the switch to operate without increasing contact resistance. Finally, we propose a physical explanation for the resistance increase, supported by related literature and experimental data.

\section{METHOD}

\section{A. Description of Switches}

All tests were performed using switches fabricated at the University of Michigan using a gold surface micromachining process. A SEM image of a typical switch is shown in Fig. 1. The switch consists of a fixed-fixed beam situated between the ground lines of a coplanar waveguide.

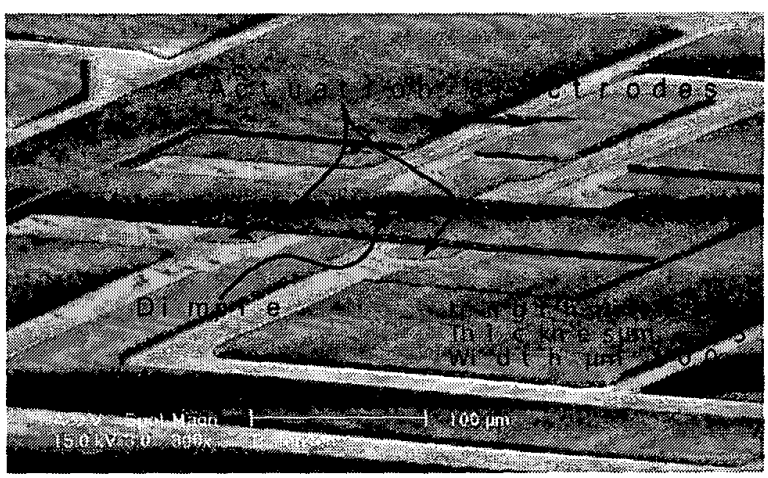

Fig. 1. SEM image of the contact-type switch

The beam and underlying electrodes are sputtered gold. Electrostatic force is used to pull the beam down until the dimple in the center of the beam contacts the central conductor of the waveguide. To avoid charging, there is no dielectric film coating the actuation electrodes. Instead, the stiffness of the beam is relied upon to prevent shorting. The total gap under the beam is $1.54 \mu \mathrm{m}$, with a dimple height of $1.18 \mu \mathrm{m}$, leaving a distance of $0.36 \mu \mathrm{m}$ to travel before contact occurs. Based on measurements of switch pull-down voltage, we estimate the Young's modulus of the gold to be $55 \mathrm{GPa}$, with a residual tensile stress of $90 \mathrm{MPa}$. Contact occurs at approximately $55 \mathrm{~V}$, and contact with the actuation electrodes occurs at about $100 \mathrm{~V}$. The beam geometry allows four-point probe measurements of contact resistance, as illustrated in Fig. 2.

\section{B. Experimental Setup}

To avoid contamination, the switches were tested in a sealed vacuum chamber kept at 5-8 mTorr. An illustration of the experimental setup is shown in Fig. 2. The vacuum pump is fitted with a foreline trap to prevent impurity diffusion into the chamber. The switches experienced no perceptible change in their behavior over a testing time of 5 months; hence, we believe that the equipment successfully prevented contamination of the chamber. The chamber is also fitted with a temperature controller which was used only to monitor temperature for the experiments described 


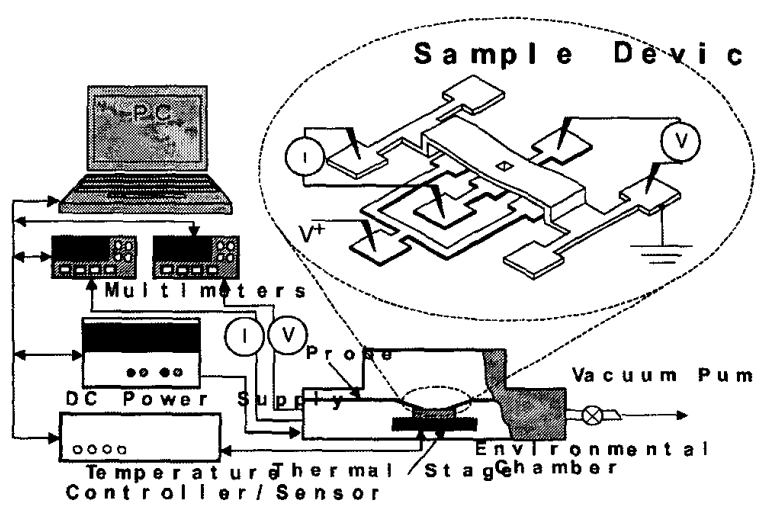

Fig. 2. Experimental Setup

here. For all experiments, the temperature remained within 3 degrees of room temperature $\left(25^{\circ} \mathrm{C}\right)$.

Contact resistance has been shown both experimentally and numerically to remain equal to its $\mathrm{DC}$ value at extremely high frequencies (for these switches, higher than $20 \mathrm{THz}$ ) [10], [11]. Hence, the experiment was simplified by measuring DC contact resistance. Two multimeters were used to record current flow and voltage drop across the contact. A dual-channel power supply was used to provide the contact current as well as the actuation signal, with a voltage amplifier to provide the high actuation voltage. All instruments were controlled by a computer running LabVIEW. The actuation voltage for all experiments described here was $72.3 \mathrm{~V}$, corresponding to a contact force of $24.0 \mu \mathrm{N}$ (based on mechanical modeling). All switches were broken in using 500 switch cycles before further testing occurred. For all tests, contact resistance was measured at each cycle as the switch opened and closed. Both hot and cold switched tests were performed.

The power supply (Agilent E3646A) allows both a current limit and a voltage limit to be set. When the output is turned on, the instrument increases the voltage until either limit is reached, allowing operation in either currentcontrolled or voltage-controlled modes. Hence, depending on the limits used, we were able to specify either the contact voltage or the contact current.

\section{Contact Theory}

The contact resistance is caused by the roughness of the contacting surfaces. As the surfaces come together, high points on each surface make contact, producing real contact at a finite number of asperities. This creates a restriction causing an enhanced resistance across the contact. Moreover, for a contact radius on the order of the electron mean free path (about $10 \mathrm{~nm}$ in gold) or smaller, the current is further constricted by boundary scattering.

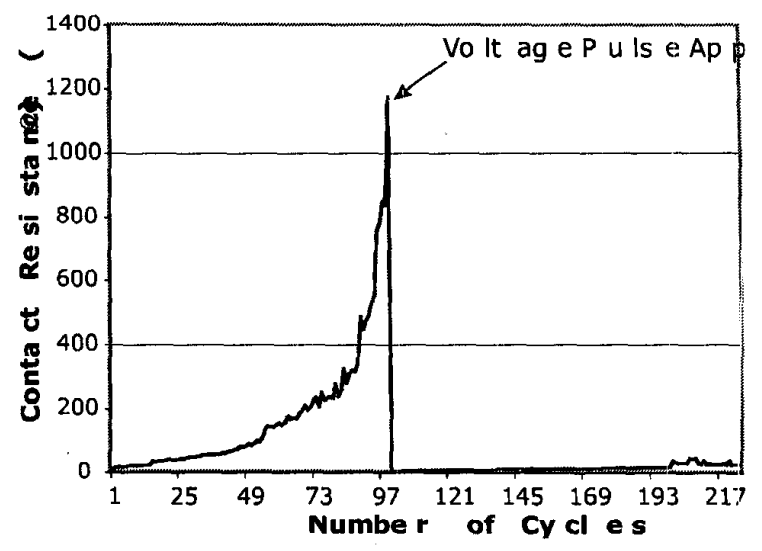

Fig. 3. Rising contact resistance leading to failure, followed by repair

For both ohmic constriction and boundary scattering, the contact resistance is approximately [12]

$$
R_{s}=\frac{1+0.83(l / a)}{1+1.33(l / a)} \frac{\rho}{2 a}+\frac{4 \rho l}{3 \pi a^{2}},
$$

where $R_{s}$ is the contact resistance, $l$ is the mean free path, $a$ is the contact radius, and $\rho$ is the electrical resistivity. It is generally assumed that the material in actual contact yields, so that, for a circular contact,

$$
a=\sqrt{\frac{F_{c}}{\pi H}}
$$

where $F_{c}$ is the contact force and $H$ is the material hardness. Note that in a real switch, several asperities will be in contact. However, (1) and (2) are convenient to use in detecting trends. In this case, $a$ represents an equivalent contact radius as if only one spot.were in contact.

As current flows through the contact, it heats the contact spot to an elevated temperature known as the supertemperature. At sufficient supertemperature, annealing or softening of the contact takes place, reducing the contact hardness and decreasing the contact resistance. For fairly low-resistance, gold contacts, this softening has been demonstrated to occur at about $80 \mathrm{mV}$, which corresponds to a supertemperature of about $100^{\circ} \mathrm{C}$ [9].

\section{Results Showing Contact Repair}

Fig. 3 shows the contact resistance of a typical switch tested using a voltage limit of $10 \mathrm{mV}$ and a current limit of $1 \mathrm{~mA}$. At the beginning of testing, the contact resistance is about $3 \Omega$, but it rapidly rises beyond the usable level (perhaps 5-10 $\Omega$ ). If allowed to continue, the resistance continues to increase until the switch on-resistance is imperceptible from the off-resistance. This behavior is consistently seen for both hot- and cold-switching at these settings of voltage and current limit. 


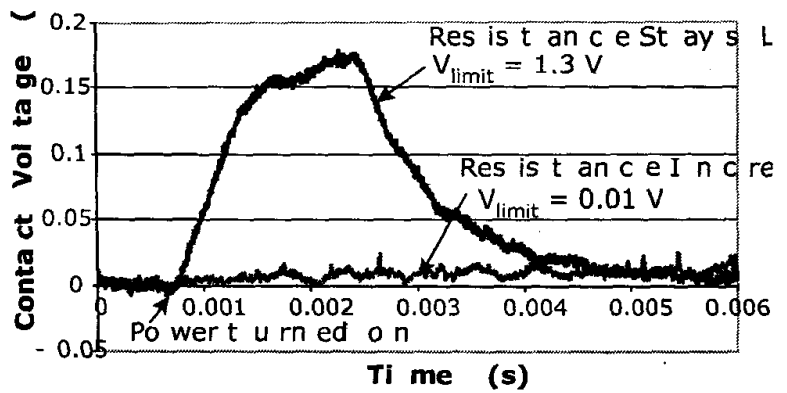

Fig. 4. Voltage trace showing annealing pulse in each cycle

\section{A. Repair of Failed Switches}

Previously, we have shown that MEMS contacts can be softened by applying a sufficiently large voltage across the contact, reducing contact resistance [9]. This occurs when the contact voltage is sufficient to raise the supertemperature high enough to anneal the contact. Using this method, we were able to repair the failed switches. In Fig. 3, a voltage pulse was applied after 100 cycles by setting the current limit to $1 \mathrm{~mA}$ and the voltage limit to $1.3 \mathrm{~V}$, which caused a rapid decrease in contact resistance. By limiting the current to $1 \mathrm{~mA}$, we allowed the power supply to adjust the voltage automatically as the resistance decreased to prevent contact melting.

We have repaired many failed switches this way. After repair, their performance is imperceptible from switches that never failed. Also, when the contact resistance is fairly low, approximately $80-100 \mathrm{mV}$ is required to soften the contact, similar to the voltage values required in [9]. However, when the contact resistance is high, a larger voltage is required to repair the contact. We believe that this is because heating in the contact is caused only by the ohmic resistance, represented by the first term in (1). For very small contact radius, the contribution of this ohmic term becomes a small fraction of the total resistance, so that a larger total voltage is required for softening. We have found that a voltage of $1.3 \mathrm{~V}$ is normally sufficient to repair even the largest contact resistance.

\section{B. Low-Resistance Switch Operation}

We can further take advantage of our knowledge of contact softening to design operating conditions which will prevent rising contact resistance. Fig. 4 shows the contact voltage measured by an oscilloscope for a single coldswitched cycle under two operating conditions. Only the first $\sim 6 \mathrm{~ms}$ after turning on the power are shown. For the higher curve, the voltage limit is set to $1.3 \mathrm{~V}$ and the current limit to $1 \mathrm{~mA}$. The measurement shows that the voltage increases initially to about $150 \mathrm{mV}$, which is high enough to anneal the contact, before decreasing to a

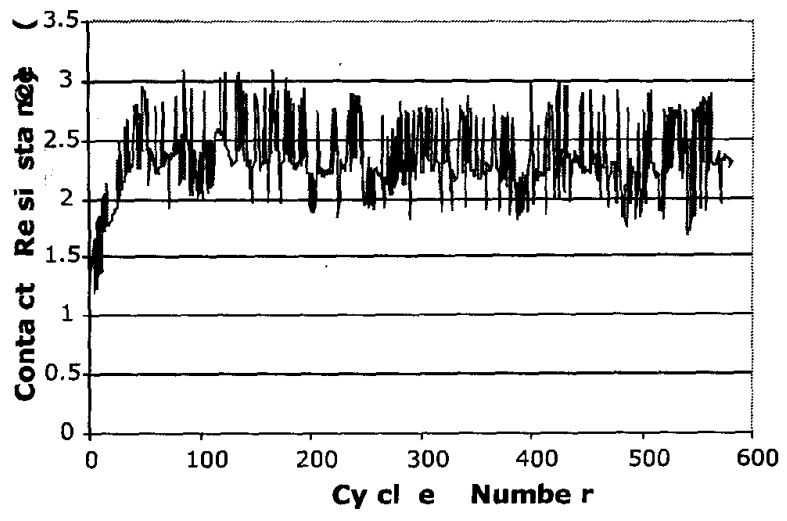

(a)

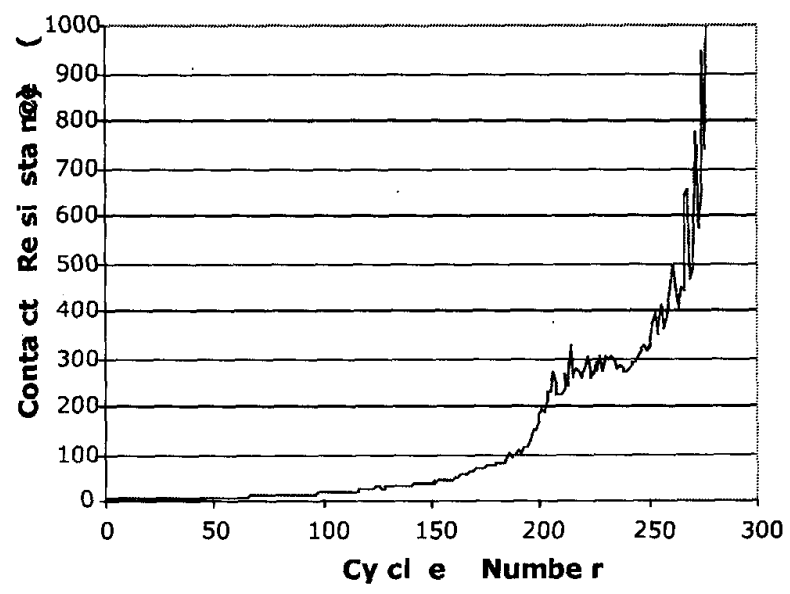

(b)

Fig. 5. Switch operation with current limit of $1 \mathrm{~mA}$ and voltage limit of (a) $1.3 \mathrm{~V}$ and (b) $0.01 \mathrm{~V}$

low operating voltage. Fig. 5(a) shows that, with annealing taking place in each switch cycle, the resistance remains low while operated using this condition. Hot-switched testing without resistance rise has also been performed. By comparison, the lower curve in Fig. 4 shows the contact voltage when the limit is reduced to $0.01 \mathrm{~V}$, so that the voltage never becomes high enough to anneal the contact. As a result, the contact resistance increases with each cycle, as shown in Fig. 5(b).

\section{Discussion of Results}

The obvious question is what could cause such a large increase in contact resistance. Based on the experimental setup and stability of measurements made over several months, we do not believe that impurities are present in sufficient numbers to consistently increase the contact resistance. In (1), only the contact radius a can change sufficiently to cause such a large resistance increase. 


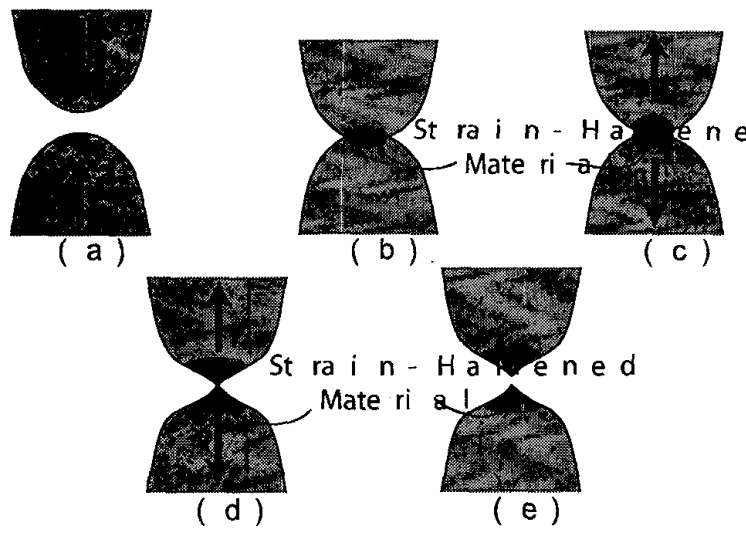

Fig. 6. Hypothesis to explain resistance rise in MEMS contact. (a): two opposing asperities are pushed together, causing plastic deformation and strain hardening (b). Further strain hardening is induced by necking of the contact spot as the surfaces are pulled apart in (c) and (d). The result is strain hardened material at the tops of each asperity (e).

Hence, based on (2), such a large increase in contact resistance must be caused by an increase in the surface hardness of the asperities.

In [13], contact of a gold STM tip with a gold substrate produced gold bumps on the substrate approximately 4$5 \mathrm{~nm}$ high. The authors explained these bumps as the products of contact necking as the tip pulled away from contact with the substrate. Such plastic deformation is normally associated with strain hardening of the material. Further, contact softening, as observed in our experiments, is explained in contact theory as the annealing of strainhardened contact surfaces [14].

Based on these observations, we have developed a hypothesis describing the source of this surface hardening, illustrated in Fig. 6. When two surfaces are forced together (a), the tops of asperities on each surface deform plastically, causing strain hardening (b). Then, as the surfaces are pulled apart, adhesion at the gold-gold interface promotes necking of the contact spot (c). This contact necking causes further strain hardening (d), leaving the tops of each asperity hardened (e). Hence, future contact force will produce a smaller contact spot due to increased hardness. This hypothesis is supported by existing literature data as well as our experiments.

\section{CONCLUSION}

This paper has studied the tendency toward contact resistance rise in metal contact RF MEMS switches under some operating conditions. Using contact heating, we were able to repair switches which had developed high contact resistance, and we demonstrated operating conditions to prevent the continual rise of contact resistance. We proposed the hypothesis that strain hardening due to contact deformation and necking is responsible for the observed increase in contact resistance. This hypothesis is supported both by our data as well as observations from existing literature. This data deepens our understanding of the nature of microscopic metal contacts and allows control of a commonly-reported switch failure mechanism, leading to improved reliability of metal contact switches.

\section{ACKNOWLEDGMENT}

This work is supported under a National Defense Science and Engineering Graduate Fellowship and by the NSF (grant no. ECS-01152222) and the CIA (grant no. 2001H605400-000).

\section{REFERENCES}

[1] S. Majumder, N.E. McGruer, P.M. Zavracky, G.G. Adams, R.H, Morrison, J. Krim, "Measurement and modeling of surface micromachined, electrostatically actuated microswitches", in Transducers 97, 1997, pp. 1145-1148.

[2] J.B. Muldavin and G.M. Rebeiz, "Inline capacitive and DC-contact MEMS Shunt Switches," IEEE Microwave and Wireless Components Letters, vol. 11, pp. 334-336, 2001.

[3] G. M. Rebeiz, RF MEMS, Theory, Design, and Technology, John Wiley \& Sons, 2003.

[4] R. Chan, R. Lesnick, D. Becher, M. Feng, "Low-actuation voltage RF MEMS shunt switch with cold switching lifetime of seven billion cycles," J. MEMS, vol. 12, pp. 713-719, Oct. 2003.

[5] B.J. Gally, C. C. Abnet, S. Brown, "Investigation of wear of microelectromechanical contacts," in Mater. Res. Soc. Symp. Proc., vol. 605 , pp. 117-122, 2000.

[6] E. J. J. Kruglick and K. S. J. Pister, "Lateral MEMS microcontact considerations," I. MEMS, vol. 8, pp. 264271, Sept. 1999.

[7] S.Majumder, N. E. McGruer, G. G. Adams, P. M. Zavracky, R. H. Morrison, and J. Krim, "Study of contacts in an electrostatically actuated microswitch," Sensors and Actuators A: Physical, vol. 93, pp. 1926, 2001.

[8] B.D. Jensen, K. Saitou, J.L. Volakis, K. Kurabayashi, "Fully integrated electrothermal multidomain modeling of RF MEMS switches," IEEE Microwave \& Wireless Comp. Letters, vol. 13, pp. 364-366, 2003

[9] B.D. Jensen, L.W. Chow, R.F. Webbink, K. Saitou, J.L. Volakis, and K. Kurabayashi, "Force Dependence of RF MEMS Switch Contact Heating," in Proc. IEEE Micro-Electro-Mechanical Systems 2004, Paper No. 111, 2004.

[10] R. Kwiatkowski, M. Vladimirescu, A. Zybura, S. Choi, "Scattering parameter model of low level electrical contacts in electromechanical microwave switches-a switch manufacturer approach," in 48th IEEE Holm Conf. On Electrical Contacts, pp. 221230, 2002.

[11] J.D. Lavers, R.S. Timsit, "Constriction resistance at high signal frequencies," IEEE Trans. Comp. Pack. Tech., vol. 25, pp. 446-452, 2002.

[12] B. Nikolić and P.B. Allen, "Electron transport through a circular constriction," Physical Review B, vol. 60, pp. 3963-3969, Aug. 1999.

[13] J.I. Pascual, J. Méndez, J. Gómez-Herrero, A.M. Baró, N. García, "Quantum Contact in Gold Nanostructures by Scanning Tunneling Microscopy," Phys. Rev. Let., vol. 71, pp. 1852-1855, Sept. 1993.

[14] R.Holm, Electric Contacts, Theory and Applications, Berlin, Germany: Springer-Verlag, 1976. 